Pierre-Alain Duc, Jonathan Braine and Elias Brinks, eds.

\title{
PAH features in Infrared Luminous Galaxies: Results from Michelle
}

\author{
M.S. Clemens \\ Università degli Studi di Padova, Italy \\ B. Nikolic, P. Alexander, G. Cotter, M.S. Longair \\ Astrophysics Group, Cavendish Lab., Cambridge, U.K.
}

\begin{abstract}
We present recent results from mid-infrared observations of a sample of nearby, infrared luminous starbursts and AGN made with the new mid-infrared instrument, "Michelle", on UKIRT. Narrow band imaging in the 7-13 micron range with sub-arcsec resolution has been used to study the spatial distribution of polycyclic aromatic hydrocarbon (PAH) features. The comparison of these sub-arcsecond resolution data with radio continuum data at similar resolution can be used to determine, 1) the sources of excitation required for PAH emission, and 2) whether $\mathrm{PAH}$ features are a measure of the relative contribution of star formation and AGN to the bolometric energy output of a galaxy. Unlike the farinfrared emission from dust, that in the mid-infrared can be used to discriminate between different heating sources.
\end{abstract}

\section{Summary}

We are observing a sample of 12 infrared luminous galaxies with narrow band imaging in the mid-IR at wavelengths of $7.9,9.7,11.6$ and $12.5 \mu \mathrm{m}$ (to be extended to 6 wavelengths with Gemini North). The $0^{\prime \prime} 7$ spatial resolution is well-matched by VLA radio data we have either observed or collected from the archive, at frequencies of $1.4,4.8,8.4$ and $15 \mathrm{GHz}$.

Of the sources observed so far Arp 157, Arp 220 and NGC 7469 show varying morphology at different mid-IR wavelengths. Arp 157 and Arp 220 show signatures of extinction even in the mid-IR, consistent with previous high estimates from optical/near-IR observations. For Arp 157 we estimate the spatial distribution of extinction by assuming that the difference between the 11.6 and $12.5 \mu \mathrm{m}$ images is due only to extinction. This simple assumption gives values consistent with a similar estimate made by comparing the free-free radio emission with a $\mathrm{Br} \gamma$ image. Both extinction estimates are consistent with the central gas column density derived from high resolution $\mathrm{CO}$ observations of the compact disk of ISM at the centre of this merger. The derived extinction map is consistent with the flux and morphology of the image at $7.9 \mu \mathrm{m}$ and no difference in the distribution of the various PAH carriers or the continuum need be invoked. Thus, at least in this source we find no evidence that PAH molecules are destroyed in any region of the young starburst environment. This is perhaps 
surprising when one considers the rather high supernova rate $\left(\sim 0.16 \mathrm{yr}^{-1}\right)$ we derive from our radio data, which is occurring in a disk approximately $500 \mathrm{pc}$ in diameter. This is an area smaller than the starburst in M 82 with a supernova rate approximately 3 times higher.

The images for NGC 7469 show a more extended structure at 7.9 and $11.6 \mu \mathrm{m}$ than at $9.7 \mu \mathrm{m}$, consistent with the existence of a starburst ring surrounding a Sy 1.2 nucleus. PAH features at 7.7 and $11.3 \mu \mathrm{m}$ contribute significantly to the flux in the starburst ring, but at $9.7 \mu \mathrm{m}$ only the continuum from the AGN is seen.

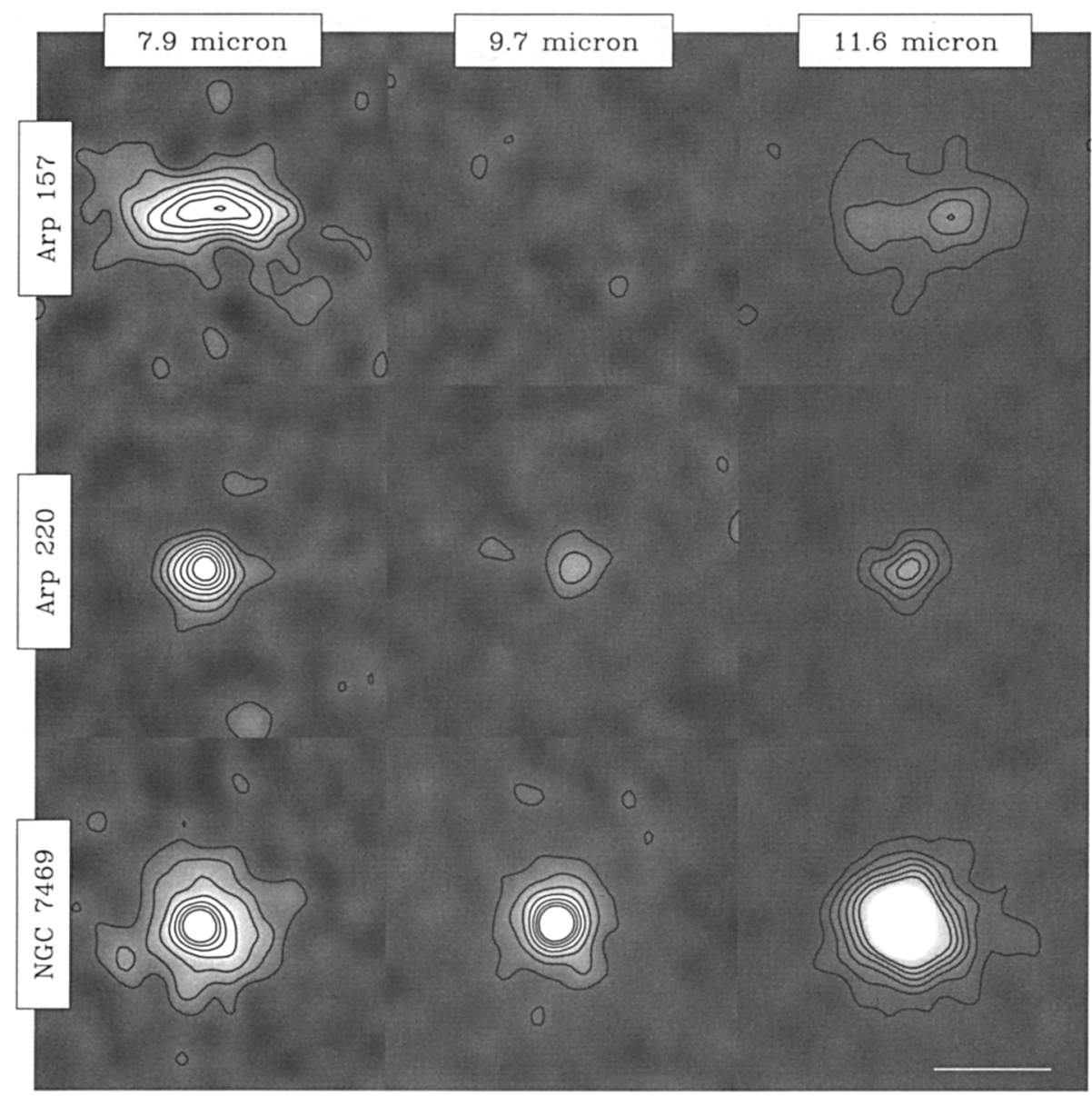

Figure 1. Narrow band images of 3 of our sample galaxies. The greyscale is the same for all the images and saturates at $0.4 \mathrm{mJy}_{\mathrm{pixel}}{ }^{-1}$. For the $7.9 \mu \mathrm{m}$ images the contours are at $(1,2, \ldots, 7) \times 10^{-4} \mathrm{Jy} \mathrm{pixel}^{-1}$, and for those at 9.7 and $11.6 \mu \mathrm{m}$ at $(0.5,1, \ldots, 3) \times 10^{-4} \mathrm{Jy} \mathrm{pixel}^{-1}$. The bar in the bottom right is $4^{\prime \prime}$ long. 